III Sección: Educación, producción y finanzas

\title{
Una pedagogía restaurativa y para la paz ante la pandemia y post pandemia: Un enfoque comunitario desde la docencia
}

Claire Marie de Mézerville López International Institute for Restorative Practices, Estados Unidos

clairedemezerville@iirp.edu https://orcid.org/0000-0002-8260-0872

Recibido: 25 de setiembre de 2020

Aceptado: 20 de noviembre de 2020

Resumen: La educación en el contexto del SARS-CoV-2 ha significado una crisis en sistemas educativos confrontados con la virtualización de emergencia, la educación a distancia o la suspensión temporal del ciclo lectivo. En algunos países se enfrenta el regreso a la presencialidad condicionada a la aplicación de protocolos preventivos. Esta emergencia global presenta inquietudes sobre cómo responder desde una pedagogía propositiva más que reactiva para dar respuesta a la situación actual y anticipar el escenario post pandemia. Este ensayo presenta las prácticas restaurativas como un abordaje que trasciende medidas disciplinarias positivas aisladas y constituye un ingrediente metodológico para una pedagogía crítica y para la paz. Una pedagogía restaurativa se consolida en la integración propositiva entre la comunidad y los aportes de la experiencia especializada. Se ofrecen implicaciones docentes y comunitarias para la respuesta presente y futura desde una ciudadanía afectiva.

Palabras clave: Prácticas restaurativas; justicia restaurativa; pedagogía; educación; docencia; pandemia; post pandemia.

\section{A restorative pedagogy for peace in the face of the pandemic and post pandemic: A community approach from teaching}

\section{(ब) $(\Theta \odot$}

La Revista Estudios es editada por la Universidad de Costa Rica y se distribuye bajo una Licencia Creative Commons Atribución-NoComercial-CompartirIgual 3.0 Costa Rica. Para más información envíe un mensaje a 
Abstract: Education in the context of SARS-CoV-2 has meant a crisis in educational systems confronted with emergency virtualization, distance education or the temporary suspension of the school year. In some countries, schools face the return to in person lessons under the enforcement of preventive protocols. This global emergency presents questions about how to respond from a propositive pedagogy and not just a reactive one, to face the current situation and anticipate the post pandemic scenery. This essay presents restorative practices as an approach that transcends disciplinary measures and constitutes a methodological ingredient for critical pedagogy and for peace. A restorative pedagogy is consolidated in the integration of community and the inputs from specialized experiences. Teaching and community implications are offered to the present and future responses from an affective citizenship perspective.

Keywords: Restorative practices; restorative justice; pedagogy; education; pandemic; post pandemic.

\section{Introducción}

Las crisis que trajo consigo o agravó la pandemia necesitan una respuesta reflexiva, relacional y comunitaria. Esto puede ilustrarse con la famosa cita de Margaret Mead, "Nunca dudes que un pequeño grupo de ciudadanos y ciudadanas reflexivos y comprometidos puede cambiar el mundo. Sin duda, es lo único que alguna vez lo ha hecho" (Herrero, 2016). Herrero (2016) va un paso más allá y menciona que falta decir explícitamente "grupo organizado de ciudadanos y ciudadanas". La realidad de los centros educativos ante la pandemia ha implicado suspensiones del curso lectivo, virtualización de emergencia, estrategias de educación a distancia y en definitiva una pérdida de la dinámica presencial acostumbrada. El objetivo de esta reflexión es describir las prácticas restaurativas como un abordaje relacional y comunitario que ofrece guías teóricas, metodológicas y valorativas para la atención a este período de transición y la incertidumbre posterior. Esta propuesta aborda de manera específica la visión de las prácticas restaurativas del International Institute for Restorative Practices (IIRP, basado en Wachtel, 2013) desde un enfoque crítico. El énfasis de este análisis se dirige a las realidades educativas presentes y dirigidas a la post pandemia, en el marco de una pedagogía restaurativa y para la paz.

\section{(c) (i) ()}

La Revista Estudios es editada por la Universidad de Costa Rica y se distribuye bajo una Licencia Creative Commons Atribución-NoComercial-CompartirIgual 3.0 Costa Rica. Para más información envíe un mensaje a 


\section{Prácticas restaurativas}

Un enfoque restaurativo es aquel que busca devolver su integridad a algo que se ha quebrantado. La palabra "restaurativo" puede resultar confusa para personas poco familiarizadas con estas propuestas y la historia de su traducción desde el inglés a partir de la justicia restaurativa en la Nueva Zelanda de los años ochentas (Gomez, Rucinski, y Higgins-D’Alessandro, 2020), podría levantar preguntas de si la traducción correcta habría sido justicia o práctica restaurativa o más bien justicia o práctica restauradora. El concepto en su forma actual se ha venido estableciendo en hispanoamérica y en desde la psicología, su pronunciación es una traducción similar al concepto acuñado por Baumrind en 1966 para describir la parentalidad autoritativa, como alternativa a parentalidades autoritarias 0 permisivas (Craig y Baucum, 2009; Feldman, 2018).

Ahora bien, ¿qué es lo que se restaura cuando se habla de prácticas restaurativas? Esta aclaración es indispensable ya que parte de una suposición: para restaurar, se entiende que algo está roto o dañado. Por ejemplo, existen profesiones que restauran piezas dentales, o restauran obras de arte y su oficio es el de ser restauradores o restauradoras. Sin embargo, al hablar de prácticas restaurativas, sin especificaciones de objeto, hay un supuesto indispensable de hacer explícito: ¿qué se restaura? Las prácticas restaurativas están orientadas a las relaciones interpersonales y comunitarias (Wachtel, 2013) en el reconocimiento de que, a nivel global, vivimos en una sociedad desigual, excluyente y deshumanizada (hooks, 1994; Haga, 2020; Sagot, 2020; Lemaitre, 2020). Estas prácticas se desarrollan a partir de la justicia restaurativa para enfocarse en comunidades educativas. En algunos contextos siguen llamándose prácticas de justicia restaurativa, aunque no estén enfocadas en aplicaciones judiciales, ya que su objetivo es restablecer la dignidad de cada persona desde un un enfoque relacional y de justicia social.

\section{(ब) (ब๑)}

La Revista Estudios es editada por la Universidad de Costa Rica y se distribuye bajo una Licencia Creative Commons Atribución-NoComercial-CompartirIgual 3.0 Costa Rica. Para más información envíe un mensaje a 


\section{Prácticas restaurativas en la educación}

Existen diferentes abordajes educativos basados en la evidencia relacionados con la respuesta al comportamiento estudiantil y la atención a la convivencia, como el Apoyo Conductual Positivo o la Educación Socioemocional (Gomez, Rucinski, y Higgins-D’Alessandro, 2020), pero las prácticas restaurativas representan una alternativa particular y complementaria a estos abordajes desde un enfoque en otorgar voz a cada integrante de la comunidad y generar espacios participativos que constituyan a la comunidad educativa en un verdadero entretejido social dinámico para el aprendizaje. Por ejemplo, al hacer referencia a las cinco dimensiones de la educación socioemocional: la auto consciencia, la consciencia social, las relaciones sociales, la toma de decisiones y la autorregulación (CASEL, 2019), es solo a través de encuentros comunitarios con una estructura metodológica intencional y flexible, orientada a animar el diálogo y horizontalizar la palabra, que la experiencia de cada estudiante será bienvenida en un ejercicio de consciencia, o de concienciación (Freire, 1985; hooks, 1994).

Asimismo, el apoyo conductual positivo cobra vida en la medida en la que exista ese encuentro relacional, que va más allá de únicamente la persona docente y la persona estudiante: incorpora el reconocimiento de que el comportamiento tiene un impacto sobre "un otro" con cuya experiencia es posible empatizar y conectar. Los diálogos restaurativos basados en preguntas no buscan nada menos que generar esos espacios afectivos, participativos y transversales, estimulando la capacidad para la toma de decisiones y resolución de problemas, dentro de una dinámica de comunidad de aprendizaje.

La equidad ha sido definida por Micah Pollock (2017) a partir de la pregunta: ¿todas y todos nuestros estudiantes tienen todas las oportunidades necesarias para alcanzar al máximo su potencial? La educación es un proceso social y de concienciación donde la atención a la conducta y a la convivencia no pueden limitarse a prevenir incidentes: es el ejercicio mismo de dignificar a la persona como sujeto, desde este enfoque de equidad. La inclusión en la educación reconoce la 
tarea aparentemente imposible de que la docencia reconozca y sobrepase con cada estudiante las diversas barreras para la educación que enfrenta cada persona. Un enfoque restaurativo reconoce que no hay motivos utilitarios en estas acciones, sino un compromiso genuino y profundo de restaurar la dignidad humana de todas las personas, visibilizando de manera particular a las y los estudiantes tradicionalmente vulnerables a ser excluidos por motivos históricos, xenofóbicos, patriarcales y estructurales. Actualmente, hay que añadir la barrera abrumadora de la distancia física y la educación a distancia.

\section{La docencia ya era difícil}

La labor de asumir la docencia como una empresa de dignificación de la persona y de construcción de sociedades más justas era de por sí un desafío abrumador, aún antes de la pandemia. Las prácticas restaurativas no son un concepto nuevo en esta tarea y las reflexiones en torno a su ejercicio se encuentran actualmente en un punto de inflexión y crecimiento (Chapman, 2020) como campo de investigación e impacto social, con nuevas exigencias, introspecciones y responsabilidades.

Si en un inicio las prácticas restaurativas fueron pensadas como una manera de aplicar justicia restaurativa en la escuela, la pandemia y la anticipación de la post pandemia han vuelto extremadamente urgente el repensar un abordaje restaurativo que no se limite al ejercicio de técnicas para el control de conducta, aún cuando este control sea aparentemente benevolente (Lustick, 2017). Es necesario cuestionar un campo de estudio que, en diferentes etapas de su desarrollo ha respondido a demandas institucionales en su mayoría bien intencionadas, pero que con su madurez como abordaje comunitario, debe establecer un enfoque ecológico que responda a las oportunidades de empoderamiento y concienciación para un involucramiento comunitario sostenible, a partir de la interdependencia entre sistemas y personas (Chapman, 2020). En la crisis sanitaria actual especialmente, las prácticas restaurativas trascienden la aplicación de reglamentos y ofrecen

La Revista Estudios es editada por la Universidad de Costa Rica y se distribuye bajo una Licencia Creative Commons Atribución-NoComercial-CompartirIgual 3.0 Costa Rica. Para más información envíe un mensaje a 
herramientas para la organización colectiva, con el fin de atender los desafíos sociales que la falta de presencialidad escolar nos plantea.

No se trata de sobrecargar a docentes y estudiantes en un tiempo de particular incertidumbre y estrés, sino por el contrario, ofrecer las herramientas relacionales y comunitarias que les permitan experimentar un sano sentido de autoeficacia y que impacte la calidad de la educación, su conexión interpersonal y su salud mental (Abrams, Hebling y Smull, 2018; Abrams, 2020). La salud mental está sufriendo de manera significativa las implicaciones de la crisis sanitaria (Orgilés, Morales, Delveccio, Mazzeschi, Espada, 2020), con un impacto especialmente grave en poblaciones de estudiantes con alto grado de vulnerabilidad social, como por ejemplo, estudiantes en confinamiento carcelario (Gagnon, 2020).

\section{Justicia Restaurativa en la escuela}

Las prácticas restaurativas pueden ser un modelo efectivo de respuesta al daño y de atención disciplinaria desde un enfoque positivo (de Mézerville, Meza, Ochoa y Ovares, 2019). En ese sentido, la justicia restaurativa en los centros escolares implica abordar la conducta con una mirada integral centrada más en el daño causado que en las reglas. Un encuentro de justicia restaurativa, en su expresión más purista, reúne a quien ha causado un daño con quienes se han visto impactados y con su comunidad, para practicar la escucha activa con respecto al impacto sobre todas las personas involucradas, fomentar la rendición de cuentas y resolver juntos y de manera colaborativa cómo enfrentar lo sucedido (Ahmed, Harris, Braithwaite y Braithwaite, 2001; Wachtel, 2013). Ahora bien, estos encuentros en sí mismos, aunque poderosos, no son suficientes: funcionan como parte de procesos comunitarios y la constitución de una cultura intencional basada en principios participativos y corresponsables por el bienestar de la comunidad (Molnar-Main, 2014).

Esto lleva a la pregunta de qué hace falta para responder al daño además de la intervención directa contra, por o con la persona que lo causó. Las otras

La Revista Estudios es editada por la Universidad de Costa Rica y se distribuye bajo una Licencia Creative Commons Atribución-NoComercial-CompartirIgual 3.0 Costa Rica. Para más información envíe un mensaje a 
dimensiones del impacto comunitario deben ser atendidas y esto aplica para la disciplina escolar, para la práctica judicial, y también en los procesos de atención al trauma: una práctica de justicia restaurativa será exitosa cuando hay espacios para el acompañamiento psicosocial, rendición de cuentas, seguimiento cercano y atención a vulnerabilidades estructurales de parte de quien causa el daño. Esto es solo un aspecto. Son indispensables también los espacios dedicados exclusivamente a acompañar a las personas más afectadas por incidentes en sus procesos de validación y sanación. La participación activa en procesos de justicia restaurativa está asociada con una reducción en los índices de síndrome por estrés post traumático, posiblemente asociados con la posibilidad de participar activamente y tener una voz en el proceso (Smith y Weatherburn, 2012).

El rol de la comunidad es igualmente fundamental y es ahí donde un enfoque restaurativo trasciende la respuesta disciplinaria y apela a la construcción de una cultura que incluye una pedagogía intencional, no solo con respecto a contenidos curriculares, sino en el proceso socializador de la vivencia escolar: los espacios de empoderamiento y participación comunitaria son indispensables para generar un clima de corresponsabilidad en los centros educativos: espacios orientados por las dimensiones de la educación socioemocional y retroalimentados por el apoyo conductual positivo, pero ejecutados a través de prácticas comunitarias y restaurativas que consoliden un sentido de pertenencia y dignidad. Necesitamos círculos para hablar sobre la vida cotidiana, sobre lo que celebramos, sobre el juego y no solo para abordar el daño y el trauma. Lo que necesita ser restaurado en las escuelas no es únicamente un incidente específico y aislado, que casi nunca lo es, sino la manera en la que interactuamos y forjamos relaciones para responder a los desafíos que plantea este momento histórico. Particularmente ante la pandemia y de cara a la post pandemia, hace falta restaurar un sentido de pertenencia en la escuela, el cual es difícil de consolidar a la distancia: las relaciones interpersonales y un sentido comunitario de la salud y el bienestar son herramientas indispensables para esto.

\section{(ब) $(\otimes \odot$}

La Revista Estudios es editada por la Universidad de Costa Rica y se distribuye bajo una Licencia Creative Commons Atribución-NoComercial-CompartirIgual 3.0 Costa Rica. Para más información envíe un mensaje a revistaestudios.eeg@ucr.ac.cr. 


\section{Apoyos teórico metodológicos de un enfoque restaurativo: una mirada crítica}

La Ventana de la Disciplina Social (Wachtel, 2013) ha sido un apoyo teórico metodológico tradicionalmente utilizado en la comprensión de las prácticas restaurativas. Una de sus adaptaciones, presentadas por VanDeering (2013) plantea la importancia de reconocer a la persona como capaz, dentro de sus propias condiciones y recursos, así como merecedora de amor y afecto: se dignifica a la persona como sujeto, en lugar de encasillarse como un objeto a ser atendido, controlado o ignorado (Ver Figura 1).

Este posicionamiento existencial y relacional en el encuentro entre docentes, estudiantes y otras personas que integran la comunidad escolar no es ni más ni menos que el espacio de la dignidad y la escucha activa: todas las personas necesitan apoyo y todas las personas son capaces de contribuir, según sus capacidades, para con el bien común. Cuestiona desde lo más básico la ilusión bancaria de la educación (Freire, 1985). Ahora bien, el distanciamiento físico hace más difíciles estos reconocimientos, los cuales lejos de volverse menos importantes, son más fundamentales que nunca. ¿Qué medios existen para identificar, expresar y acercarse a la realidad compartida de una educación atravesada por la incertidumbre, las limitaciones y los riesgos sociales, de salud e inclusive políticos que trajo consigo la pandemia? ¿Qué posibilidades existen para, desde la docencia a distancia, transmitir al estudiantado que son capaces de aprender, que seguimos estando dispuestas y dispuestos a desafiarlos en su aprendizaje y a estimularles para desarrollarse y crecer? 


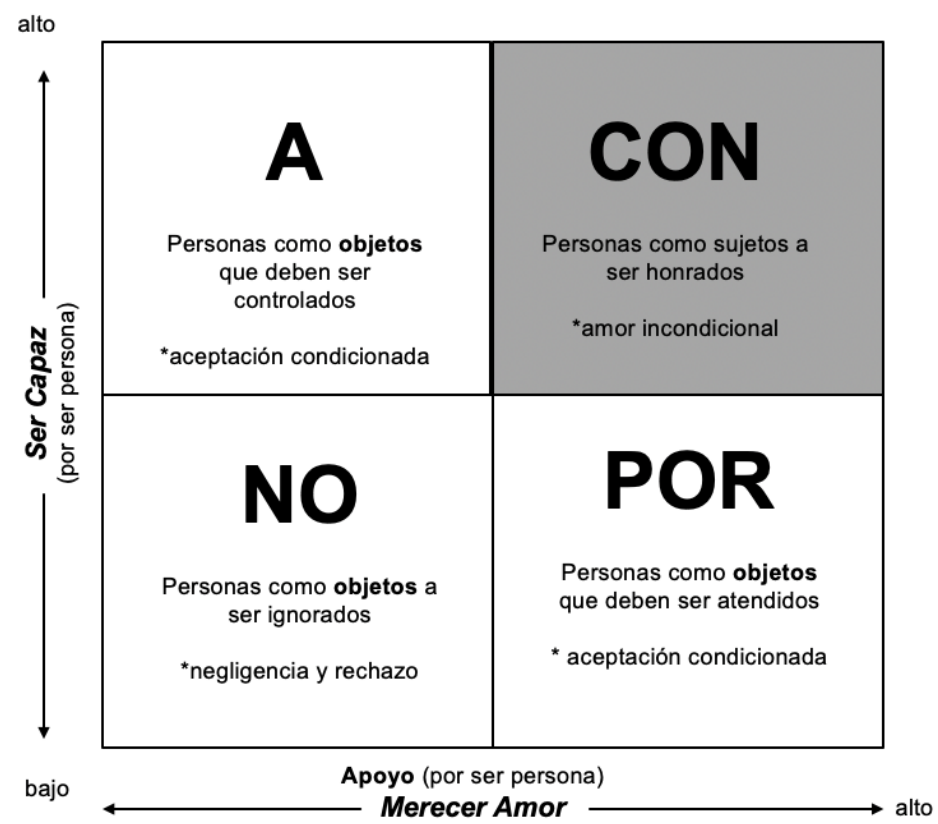

Figura 1. Ventana Relacional, según influencias de Simon y Kohn. Vandeering, D. (2013). A window on relationships: reflecting critically on a current restorative justice theory. Restorative Justice: An International Journal. 1(3), 311-331

Un enfoque restaurativo tradicionalmente se ha apoyado en los principios del proceso justo de Kim y Mauborgne (2003) sobre cómo toda toma de decisiones y cambio comunitario escolar debe ser participativo, explicado y con claridad de expectativas. Estos principios evidentemente también podrían utilizarse con miras a apaciguar a las comunidades para que se adhieran a lo que indique la voz adultocéntrica, utilizando un abordaje restaurativo como una manera de sostener lo tradicional y de mantener un autoritarismo "benevolente" (Lustick, 2017; Chapman, 2020). En ese sentido, las prácticas restaurativas podrían volverse un enfoque tentador para controlar y disciplinar con amabilidad, pero dejando de lado una dimensión dignificadora de abrir espacios reflexivos y transformadores en la educación. La participación, la explicación y la claridad de expectativas podrían, por otro lado, conceptualizarse como escalones hacia esa pedagogía crítica (Freire,1985; hooks, 1994), como un espacio liberador en el que el conocimiento no se deposita, sino se construye en conjunto.

\section{(c) (†) (-)}

La Revista Estudios es editada por la Universidad de Costa Rica y se distribuye bajo una Licencia Creative Commons Atribución-NoComercial-CompartirIgual 3.0 Costa Rica. Para más información envíe un mensaje a revistaestudios.eeg@ucr.ac.cr. 
Los tres principios antes mencionados, más que aproximarnos a procesos justos de una jerarquía benevolente, nos ofrecen la posibilidad de llevarnos a recuperar un sentido reflexivo y activo de pertenencia y confianza comunitaria mediante la participación empoderada y la escucha activa (principio uno: participación), la transparencia y la rendición de cuentas (principio dos: explicación) y los ciclos constantes de retroalimentación con miras a la corresponsabilidad (principio tres: claridad de expectativas). Actualmente, necesitamos renovar esa noción que más allá de ser constructivista, debe ofrecerse como transformadora de la realidad, ya que los desafíos del mundo abatido por el COVID19 requieren de un involucramiento compartido y comprometido con el bien común (de Mezerville, 2020). Esto es imposible si no existe confianza comunitaria.

Los centros educativos tienen el rol privilegiado de hacer converger a estudiantes menores de edad y adultos, sus familias y posiblemente los gobiernos locales. ¿Cuáles son los diálogos intercomunitarios que pueden facilitarse desde la escuela? (de Mezerville, 2020; Abrams, 2020).

De ahí que entendemos las prácticas restaurativas, no meramente como esa respuesta disciplinaria ante una falta a un reglamento de evaluación de los aprendizajes, sino como una construcción comunitaria y relacional en la que la mayor parte de la creatividad, energía y motivación se dirija a las aproximaciones que fomenten relaciones fuertes, sentido de pertenencia y participación solidaria. Ciertas prácticas, como las que implementa el IIRP (Augustine, Engberg, Grimm, Lee, Lin, Christianson y Joseph, 2018), ofrecen abordajes metodológicos específicos que han probado tener efectividad, pero que no son fines en sí mismos, sino medios para una comunidad relacional más fuerte.

\footnotetext{
(c) (1)(2)

La Revista Estudios es editada por la Universidad de Costa Rica y se distribuye bajo una Licencia Creative Commons Atribución-NoComercial-CompartirIgual 3.0 Costa Rica. Para más información envíe un mensaje a revistaestudios.eeg@ucr.ac.cr.
} 


\section{Implementación Escolar: Errores y Aprendizajes}

El IIRP ha desarrollado un modelo de formación y desarrollo profesional en centros educativos, con más de veinte años de experiencia. En el año 2018 se desarrolló el primer estudio aleatorizado con 54 centros educativos públicos en Pittsburgh, Estados Unidos, donde la mitad de los centros educativos llevaron un proceso de implementación de prácticas restaurativas durante dos años, facilitado por el IIRP y los otros centros educativos fueron de control. Se encontraron diferencias significativas en la mejora de relaciones docentes-estudiantes, una reducción en las suspensiones y expulsiones y una disminución en la brecha racial en las medidas disciplinarias, debido a la menor pérdida de lecciones por parte del estudiantado afroamericano (Augustine, et al., 2018).

Este estudio reflejó también diferencias importantes en el nivel de efectividad de las prácticas restaurativas en preescolar y primaria en comparación con secundaria. En secundaria, los resultados fueron menos eficaces, lo cual plantea la pregunta de si el modelo de implementación del IIRP manifiesta un sesgo adultocéntrico: quizás en la medida en la que el estudiantado llega a la adolescencia se vuelve notoria la importancia de su propio involucramiento y participación en estos procesos, en lugar de ser solo beneficiarios pasivos de los mismos (de Mezerville, 2019).

Existen más preguntas pendientes para aproximarnos a una pedagogía restaurativa, descolonizadora, culturalmente apreciativa y comunitariamente consciente de la sabiduría contextual necesaria para enfrentar los desafíos globales. ¿Qué tanto nos hace falta caminar con las comunidades escolares incluyendo a los liderazgos juveniles en la construcción de una pedagogía para la paz? ¿Qué tanto se ofrecen herramientas para la escucha activa, la noviolencia, la mediación y la participación para esos liderazgos?

La implementación de prácticas restaurativas necesita partir de diálogos comunitarios e intercomunitarios en lugar de implementaciones impuestas. Hay personal educativo que puede sentir que el proyecto o programa de prácticas 
restaurativas se le impone de manera punitiva por parte de autoridades centralizadas o que solo funciona si es ejecutado por consultores externos que "saben más que ellas y ellos". Esos abordajes colonizadores y paternalistas son insostenibles y nos alejan de una verdadera pedagogía para la paz.

Existen conversaciones indispensables para desarrollar estos procesos:

1. ¿Existe una comprensión reflexiva del enfoque restaurativo antes de "lanzarlo"?

2. ¿Se alinea con otras iniciativas de la escuela y con sus buenas prácticas ya existentes? ¿Las autoridades de los ministerios de educación tienen consciencia de estas buenas prácticas y la capacidad de desarrollar procesos dialógicos para la implementación reflexiva?

3. ¿Hay disposición del personal para involucrarse activamente en un proceso de transformación sociocultural en la manera de atender el cambio, las faltas y las crisis?

4. ¿Existe una comprensión genuina del compromiso que esta implementación involucra?

5. ¿Cuáles son los liderazgos comunitarios y las buenas prácticas ya existentes que deben ser honradas?

Estas preguntas indispensables confirman el desafío que plantea hooks al afirmar que la educación liberadora no puede limitarse a un conocimiento liberador, sino a una pedagogía liberadora (hooks, 1994). Este abordaje no se limita a "liberar" al estudiantado desde un enfoque asistencialista: la pedagogía liberadora también funciona de manera dinámica en la incorporación humanista de la comunidad docente, desde un acompañamiento culturalmente respetuoso, socialmente comprometido y robusto en generar espacios de escucha y apoyo.

\section{(c) (i) (2)}

La Revista Estudios es editada por la Universidad de Costa Rica y se distribuye bajo una Licencia Creative Commons Atribución-NoComercial-CompartirIgual 3.0 Costa Rica. Para más información envíe un mensaje a 


\section{Sabiduría Contextual}

Se hace fundamental reconocer la importancia de descolonizar un enfoque que no puede ser globalizado y unitalla para forzarse a lo local: una pedagogía restaurativa y para la paz se informa de referentes internacionales y experiencias diversas, se nutre de metodologías y evidencias con el propósito de interactuar de manera creativa y respetuosa con la sabiduría contextual de cada comunidad. Esa sabiduría contextual se activa solo cuando de manera intencional abrimos espacios de diálogo valientes, participativos y equitativos. Eso a veces nos confronta con la necesidad de salir de zonas de comodidad y de enfrentar problemas históricos y latentes. Es válido necesitar apoyo, ya que estos procesos no son sencillos. Responder a los desafíos del escenario que nos presenta la pandemia ya de por sí es sumamente complejo: "Aquí la hipótesis es que el diálogo entre profesionales con competencias específicas y una comunidad de cuido con sabiduría contextual, donde es ésta última la que toma las decisiones finales, es lo que resultará en mejores decisiones que la sola discreción de profesionales" (Ahmed, Harris, Braithwaite and Braithwaite, 2001, p. 64).

No podemos perder la visión sistémica de toda comunidad escolar. En el esquema ecológico de Bronfenbrenner (Craig y Baucum, 2009) la persona estudiante, así como la persona docente, está rodeada por su microsistema familiar, su mesosistema de interacciones entre la escuela, el hogar, el barrio, la iglesia y un sistema de medios de comunicación y en el que debemos incorporar la variable de las redes sociales (Oberst, Chamarro y Renau, 2016). Todo esto inmerso en una cultura y contexto histórico. A todo lo anterior, la pandemia presenta un escenario aún más complejo para esta generación.

Una práctica restaurativa que no sea consciente de un enfoque sistémico, histórico y ecológico podría más bien ser una herramienta para meramente mantener el orden (Lustick, 2017) a costa de la complejidad comunitaria y de la individualidad de estudiantes cuya conducta no está aislada: está directamente asociada con condiciones de desigualdad que harán muy probable su exclusión del 
sistema educativo. Una pedagogía restaurativa reconoce la necesidad de hablar intencionalmente sobre estas fuerzas excluyentes que se mueven en nuestras sociedades, manifiestas, entre otros fenómenos, a través del camino de la escuela a la cárcel o corredor de escuela a prisión (de Mezerville, et al., 2019).

Es necesario abordar estos temas desde una pedagogía crítica, participativa y comprometida con involucrar a la comunidad en estas situaciones de fondo. Un modelo reflexivo de justicia restaurativa crítica propone prácticas en las que el estudiantado no se limite a reflexionar sobre su conducta: se promueve el reconocimiento activo y reflexivo sobre las relaciones entre su comportamiento, su comunidad escolar, el contexto histórico y el sistema más amplio en el que coexiste (Knight y Wadhwa, 2014). ¿Qué podría ser más educativo que esto?

Esto es ahora más necesario que nunca, ya que los riesgos de exclusión se ven agravados por las implicaciones que la educación a distancia ha generado, desapegando a la juventud de los sistemas de protección y resiliencia que ofrece la asistencia física al centro educativo. La separación física de las y los menores de edad de la escuela se suma al trauma colectivo que el distanciamiento solidario conlleva. Si bien el distanciamiento es efectivamente solidario, ya que la salud es corresponsabilidad de todas las personas, el impacto sobre la desigualdad social y el desarrollo de niñas, niños y adolescentes (Gagnon, 2020) nos presenta desafíos muy serios.

\section{¿Cómo nos preparamos, desde la educación, para el mundo post pandemia?}

La pandemia dejará una sombra longeva. La amenaza del SARS-CoV-2 está lejos de desaparecer, pero aún cuando eso suceda -y si sucede-, sus secuelas serán fantasmas por enfrentar por mucho tiempo más. Una pedagogía restaurativa y para la paz no solo será necesaria para reconstruir una presencialidad educativa. Es urgente ahora, en medio del distanciamiento. No se trata de una única metodología específica: no es únicamente hacer un círculo virtual aislado o tener diálogos restaurativos por teléfono basados en una guía metodológica de 
preguntas, aunque estaría muy bien hacer eso. Las prácticas restaurativas son medios y no fines en sí mismos: el objetivo es la cohesión relacional, reflexiva y comunitaria. La tarea ineludible consiste en generar iniciativas orientadas a comunidades educativas comprometidas con una ciudadanía afectiva, aún en medio de la distancia física.

Incorporar los afectos en la educación puede generar mucha resistencia y temor en las personas adultas que trabajamos en la educación. Una pedagogía restaurativa y para la paz necesita regresar a sus fundamentos más esenciales desde la pedagogía crítica. El empoderamiento no puede ocurrir si nos rehusamos a ser vulnerables mientras a la vez pedimos a las y los estudiantes que tomen riesgos y participen (hooks, 1994). Igualmente, la auto actualización docente no es una tarea individualizada y privada: es un desafío que requiere de altas capacidades y de mucho apoyo. Es una tarea comunitaria y ciudadana así como una prioridad actual para sobrellevar la presión laboral y la incertidumbre social que la pandemia ha traído consigo. La comunidad de apoyo para docentes mediante la apertura de espacios de reflexión e intercambio de experiencias entre los docentes mismos es absolutamente imprescindible para que el aula pueda eventualmente consolidarse como un lugar apropiado para la contención, participación y apoyo para el estudiantado.

La docencia a través de plataformas virtuales, mediante el intercambio de materiales a distancia, e inclusive en el escenario de una suspensión temporal del año lectivo también puede incorporar una pedagogía restaurativa al procurar un contacto directo docente-estudiante, ya sea por videollamada, por llamada telefónica o por envío de cartas. Un círculo virtual al inicio de una clase en línea para saludar a cada estudiante y llamarle por su nombre; una nota de ánimo en el paquete de fotocopias enviadas, una llamada telefónica solo para saludar al niño o niña, ofrece un sentido de pertenencia y de significancia: queremos que la persona menor de edad pueda decir "sigo siendo alguien en la escuela" (George, 2014). Esa identidad no necesita estar asociada a un uniforme o al espacio físico: consiste en 
reconocerse en la mirada de otra persona que valida su participación e individualidad en una relación afectiva. La docencia nos ofrece la oportunidad para hacer justamente eso, especialmente en medio de esta crisis.

Asimismo, es necesario fortalecer los liderazgos comunitarios que en los centros educativos incluyan a docentes, familias y liderazgos estudiantiles, que fomenten comunicaciones interpersonales e intercomunitarias, como por ejemplo, crear inter- comunidad con las comunidades docente y familias, ofreciendo protagonismo y participación activa niños, niñas y adolescentes (de Mezerville, 2019). El movimiento internacional por la justicia y las prácticas restaurativas ha desarrollado experiencias y metodologías específicas que podemos explorar de manera participativa desde la propia comunidad escolar, acercándonos a personas con conocimiento especializado o guías teórico-metodológicas y sobre todo, dándole al liderazgo comunitario el protagonismo de las decisiones que respondan de forma integral a las necesidades de las personas por un mayor sentido de pertenencia (Abrams, 2020).

Estas construcciones serán fuertes si se retroalimentan de valores como la noviolencia y la participación ciudadana para enfrentar el cambio, la crisis y el conflicto. La noviolencia es más efectiva en la medida en la que incorpora entrenamiento, estructura metodológica y relaciones fuertes (Haga, 2020). No podemos dejar de lado la necesidad de entrenamiento, la retroalimentación que nos ofrecen modelos basados en la evidencia, ni los apoyos interdisciplinarios respaldados por las ciencias sociales. Las situaciones que estamos enfrentando en la virtualidad y la educación a distancia, así como en el futuro reencuentro, no son sencillas y tener enfoques sensibles, reflexivos e informados es indispensable. No obstante, descansar únicamente en los criterios de expertos no permitirá generar el entretejido social necesario para sanar. Cada centro educativo necesitará trabajar como una comunidad fuerte que ofrezca apoyo, que reflexione, que involucre la voz de niños, niñas y jóvenes a través de círculos, diálogos y preguntas para asumir el

\section{(c) (i) (2)}

La Revista Estudios es editada por la Universidad de Costa Rica y se distribuye bajo una Licencia Creative Commons Atribución-NoComercial-CompartirIgual 3.0 Costa Rica. Para más información envíe un mensaje a revistaestudios.eeg@ucr.ac.cr. 
desafío de transformar la realidad. Los modelos más informados de capacitación e implementación de prácticas restaurativas deben incorporar esta consciencia.

\section{¿Cómo pueden ayudar las prácticas restaurativas?}

Se trata de abordar la incertidumbre en conjunto. Existe un dicho: "si me das un pez, me alimentaste un día. Si me enseñas a pescar, me alimentaste hasta que el río se contamine o invadan la bahía para un desarrollo industrial. Pero si me enseñas a organizarme con mis pares, entonces, no importa el desafío, me sabré unir con ellas y ellos y diseñaremos nuestra propia solución”. (Levins Morales, 2020). Las prácticas restaurativas pueden ayudar a través de:

1. Relaciones interpersonales y conexiones intercomunitarias: las prácticas restaurativas ofrecen un aporte significativo a la salud comunitaria, ya que las relaciones fuertes mejoran nuestro sentido de eficacia, de comunidad y de pertenencia que se sensibiliza a la corresponsabilidad por el bien común. La salud física y mental es responsabilidad de todos: este año nos ha enseñado esto.

2. Animando la autoactualización en la comunidad docente de manera comunitaria y no únicamente impuesta. A menudo hay mucha resistencia docente (Blood y Thorsborne, 2006) ante la implementación de un nuevo programa, por más "bueno" que ese programa sea. Lejos de ver esa resistencia como un obstáculo, deberíamos considerar esta realidad como una invitación aún más vehemente a generar círculos de escucha activa y empática con las personas y a generar estrategias creativas de trabajo conjunto.

3. Ofreciendo estructura metodológica y experiencia basada en la evidencia desde un enfoque relacional y comunitario.

4. Mejorando el sentido de pertenencia, que incrementa la probabilidad de actuar para promover el bienestar común (Abrams, 2020), ya que aunque sabemos que niños, niñas y adolescentes quizás sean 
inmaduros en su desarrollo socioemocional, estas prácticas generan esa Zona de Desarrollo Próximo de la que hablaba Vigotsky: el aprendizaje socioemocional y la ciudadanía afectiva no se enseñan siguiendo un currículum o ciertos pasos metodológicos: se enseñan en un contexto relacional.

\section{En conclusión}

Este momento histórico nos encara con una población estudiantil que necesita navegar el trauma individual y colectivo. No hay respuestas sencillas. Necesitamos reconocer la importancia de la resiliencia a partir de la experiencia escolar (Henderson y Milstein, 2003). Sanar emocionalmente no puede aislarse a los espacios clínicos: sí existe una necesidad de atención psicosocial especializada y de vinculación interinstitucional entre educación y salud, pero los abordajes educativos necesitan estar preparados para responder de manera apropiada e integral a las condiciones personales y sociales relacionadas con los traumas que ya estamos enfrentando.

Este proceso de recuperación y de reconstrucción de una dinámica educativa con la infancia y adolescencia no debe partir de un asistencialismo adultocéntrico, sino de un enfoque sistémico que incluya familias, gobiernos locales, y a la juventud misma: la esperanza está en reconocer la necesidad urgente de comunidades orientadas a una ciudadanía afectiva basada en la escucha activa, la comunicación no violenta, la empatía y abordajes humanistas que nos ayuden a navegar la polarización social.

Finalmente, podremos desarrollar programas llamativos y tecnología de punta, pero lo que niños, niñas, jóvenes, docentes, familias y demás integrantes de nuestras comunidades necesitan para enfrentar la pandemia y la post pandemia es un sentido de empoderamiento, radicado en una ciudadanía organizada, reflexiva y afectiva. Una pedagogía restaurativa y para la paz es un camino que puede

\section{(ब) $(\Theta \odot$}

La Revista Estudios es editada por la Universidad de Costa Rica y se distribuye bajo una Licencia Creative Commons Atribución-NoComercial-CompartirIgual 3.0 Costa Rica. Para más información envíe un mensaje a 
devolvernos la conexión con lo mejor de nosotras y nosotros mismos en este proceso para sostener, reconstruir y sanar.

\section{Referencias}

Abrams, G.B. (2020). Salud Comunitaria y Prácticas Restaurativas. [Website]. Recuperado de: https://la.iirp.edu/2020/05/01/salud-comunitaria-y-practicasrestaurativas/

Abrams, G. B., Hebling, M. J. \& Smull, E. (2018, Octubre). Proactive restorative practices: Creating the conditions for individuals and communities to flourish. Ponencia presentada en Strengthening the Spirit of Community, IIRP World Conference, Detroit, MI.

Ahmed, E., Harris, N., Braithwaite, J. \& Braithwaite, V. (2001). Shame Management Through Reintegration. Cambridge Criminology Series.

Augustine, C., Engberg, J., Grimm, G. E., Lee, E., Lin, E., Christianson, K., Joseph, A. (2018). Can Restorative Practices Improve School Climate and Curb Suspensions? An Evaluation of the Impact of Restorative Practices in a MidSized Urban School District. Santa Monica, CA: RAND Corporation.

Blood, P. \& Thorsborne, M. (2006). Overcoming resistance to whole-school uptake of restorative practices. Congreso 8th International Conference on Conferencing, Circles and Other Restorative Practices, Estados Unidos.

CASEL (2019). What is SEL? [Website]. Recuperado de: https://casel.org/whatissel/

Chapman, T. (2020). A Vision for Restorative Justice: From Ego to Ecosystem. [Video de YouTube]: https://youtu.be/T5P6THqN8I4

Craig, G. \& Baucum, J. (2009). Desarrollo Psicológico. 9na. Edición. Pearson.

De Mezerville López, C. (2019). Protagonismo Adolescente. El rol del estudiantado en la implementación de Prácticas Restaurativas en el Liceo San Rafael de Alajuela. Revista de Educación y Desarrollo, 50, 9-17.

\section{(c) (i) (2)}

La Revista Estudios es editada por la Universidad de Costa Rica y se distribuye bajo una Licencia Creative Commons Atribución-NoComercial-Compartirlgual 3.0 Costa Rica. Para más información envíe un mensaje a revistaestudios.eeg@ucr.ac.cr. 
De Mézerville López, C., Meza Rodríguez, A., Ochoa, T. A., \& Ovares Fernández, Y. (2019). APLICACIONES EDUCATIVAS EN SECUNDARIA PARA LA PREVENCIÓN DE LA CRIMINALIZACIÓN ESTUDIANTIL: UN ENFOQUE DESDE LA JUSTICIA RESTAURATIVA Y LOS DERECHOS HUMANOS. Revista Latinoamericana De Derechos Humanos, 30(1), 123-148. https://doi.org/10.15359/rldh.30-1.5

De Mezerville López, C. (2020). El desafío del diálogo intercomunitario. [Website]. Recuperado de: https://la.iirp.edu/2020/07/30/la-esperanza-esta-en-eldialogo-intercomunitario/

Feldman, R.(2018). Psicología con Aplicaciones de América Latina. 12Va Edición. McGrawHill.

Freire, P. (1985): Pedagogía del oprimido. Siglo XXI Editores

Gagnon, J.C. (2020). The solitary confinement of incarcerated American youth during COVID-19. Psychiatry Res. 292, 113219. doi: 10.1016/j.psychres.2020.113219

George, G. (2014) Affect and emotion in a Restorative School. In Kelly, V. and Thorsborne, M. (Eds.), The Psychology of Emotion in Restorative Practice (pp. 200 - 233). Jessica Kingsley Publishers.

Gomez, J. A., Rucinski, C. L., \& Higgins-D’Alessandro, A. (2020). Promising pathways from school restorative practices to educational equity. Journal of Moral Education, doi: 10.1080/03057240.2020.1793742

Haga, K. (2020). Healing Resistance. Parallax Press.

hooks, bell (1994). Teaching to Transgress. Routledge.

Henderson, V. Milstein, M. (2003). Resiliencia en la Escuela. Buenos Aires: Paidós. Kim, W.C., \& Mauborgne, R. (2003). Fair Process. Managing in the Knowledge Economy. Harvard Business Review. The Best of HBR, 127-136.

Knight, D. and Wadhwa, A. (2014). Expanding Opportunity through Critical Restorative Justice Portraits of Resilience at the Individual and School Level. Studies in Education, 11(1), 11-33.

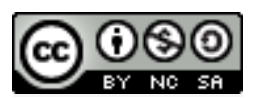

La Revista Estudios es editada por la Universidad de Costa Rica y se distribuye bajo una Licencia Creative Commons Atribución-NoComercial-CompartirIgual 3.0 Costa Rica. Para más información envíe un mensaje a revistaestudios.eeg@ucr.ac.cr. 
Leandro Herrero (2016, 19 de febrero). The missing word in the famous Margaret Mead quote. https://leandroherrero.com/the-missing-word-in-the-famousmargaret-mead-quote/

Lemaitre, Julieta. (2020). Transitional justice and the challenges of a feminist peace. 1•CON. 18(2), 455-460. doi:10.1093/icon/moaa050

Levins Morales, R. (2020). Give me a fish. [Website]. Recuperado de: https://www.rlmartstudio.com/product/give-me-a-fish/

Liang, C.T., Rocchino,G.H., Gutekunst, M.H.C., Cléopatre Paulvin, C., Li, K.M. \& Elam-Snowden, T. (2019). Perspectives of Respect, Teacher-Student Relationships, and School Climate Among Boys of Color: A Multifocus Group Study. Psychology of Men \& Masculinities, 21(3), 345-356. http://dx.doi.org/10.1037/men0000239

Lustick, Hillary (2017). "Restorative Justice" or Restoring Order? Restorative School Discipline Practices in Urban Public Schools. Urban Education, 00(0), 1-28. doi: $10.1177 / 0042085917741725$

Molnar-Main, S. (2014). Integrando Prevención del Acoso Escolar con Prácticas Restaurativas en Instituciones Educativas. Consideraciones para Practicantes y Gestores de Políticas. Clemson University.

Oberst, U., Chamarro, A. \& Renau, V. (2016). Gender Stereotypes 2.0: Selfrepresentations of Adolescents on Facebook. Revista Científica de Educomunicación, 24(48), 81-90.

Orgilés, Mireia; Morales, Alexandra; Delveccio, Eliza; Mazzeschi, Claudia; Espada, José P. (2020). Immediate psychological effects of the COVID-19 quarantine in youth from Italy and Spain. Ministry of Science and Innovation of Spain and the European Regional Development Fund (PSI2017-85493-P).

Pollock, M. (2017). Schooltalk: Rethinking What We Say Aboutand ToStudents Every Day. The New Press.

Sagot, M. (2020). Violence Against Women: Contributions from Latin America. The Oxford Hadbook of the Sociology of Latin America. doi: 
10.1093/oxfordhb/9780190926557.013.32

Smith, N. \& Weatherburn, D. (2012). Youth justice conferences versus children's court: A comparison of re-offending. Crime and Justice Bulletin. 160.

Vandeering, D. (2013). A window on relationships: reflecting critically on a current restorative justice theory. Restorative Justice: An International Journal. 1(3), 311-331

Wachtel, T. (2013). Defining Restorative. [Archivo en PDF]: https://www.iirp.edu/pdf/Defining-Restorative.pdf 